\title{
Intelligent Condition Monitoring System (ICMS) for Unmanned Air Vehicle (UAV), Unmanned Surface Vehicle (USV) and Autonomous Underwater Vehicle (AUV), Robots: A Feasibility Study
}

\author{
${\underline{\text { Xiang } X \mathbf{u}^{\mathrm{a}}}}^{\text {and Amir Anvar }}{ }^{\mathrm{a}}$ \\ ${ }^{a}$ School of Mechanical Engineering, Faculty of Engineering, Computer, and Mathematical Science, \\ The University of Adelaide, South Australia, Australia \\ Emails: a1600607@student.adelaide.edu.au, amir.anvar@adelaide.edu.au
}

\begin{abstract}
An Intelligent Condition Monitoring System (ICMS) is important for different types of vehicles used to perform unmanned tasks. The main function of the ICMS is to ensure that the unmanned vehicle is in robust condition. In this paper, three main sections are built in ICMS. That is, structure health monitoring, power management and speed detection. For the structure monitoring, the pressure distribution of the vehicle is obtained by using CFD modelling. CFD is fluent software that contains the broad physical modelling capabilities needed to model flow, turbulence and heat transfer ranging from air flow over an aircraft wing to combustion in a furnace. In the test, the vehicle is required to work in regular condition, and the pressure distribution is obtained. The pressure distribution graph indicates the pressure at each part of the structure. Then, the part of the structure that needs to be monitored is determined. In relation to power management, the DS2788 as a battery chip constantly monitors the status of multiple cell batteries. These include the current, voltage, temperature and available capacity. It is applied for the detection remaining power in the battery. For the speed detection part, the RPM sensors are used to detect speed in real time and to make sure that the vehicle is running in the speed expected.
\end{abstract}

Keywords: Intelligent, Sensors, Status Monitoring, Structure detection, Power Management, Speed Detection 
X.Xu, A.Anvar, Intelligent Condition Monitoring System (ICMS) for Unmanned Air Vehicle (UAV), Unmanned Surface Vehicle (USV) and Autonomous Underwater Vehicle (AUV), Robots.

\section{INTRODUCTION}

A considerable amount of research is devoted to develop Intelligent Condition Monitoring System that achieves the function of structure detection. The principle of structure detection is to analyze mode shape curvatures and modal parameters (natural frequency \& modal damping) by using sensors to assess the health state of a structure. Any existing damage leads to change in the modal properties in structure that can be obtained as a set of data through sensors. However, due to noise including in test environment, the curvatures are inaccurate. Therefore, it is required to obtain accurate curvatures which precisely indicate the status of a structure. We used the following approach to obtain the data of a structure.

1. The method which applies vibration sensors to detect the natural frequency of the wings.(Tao D 2012)

This method provides an accurate model of an AUV's wing. By building vibration sensors onto the wing, the data and the curvatures of the structure can be collected. However, due to the noise of the environment, the data gathered is not accurate. In order to obtain accurate data and curvatures, more filters should be used in the ICMS,

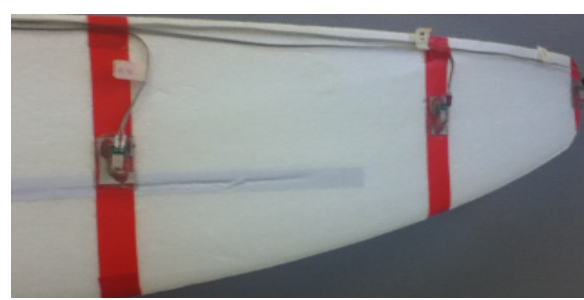

Figure 1.AUV's wing built with vibration sensors which makes the system more complex.

Figure2 has shown the pressure distribution of the AUV's wings. Sensors will be built on the location where the pressure is relatively high (Yellow part indicates high pressure). Because, the part of the wings that experience high pressure have high probability of fracture.

By applying CFD software, a simple

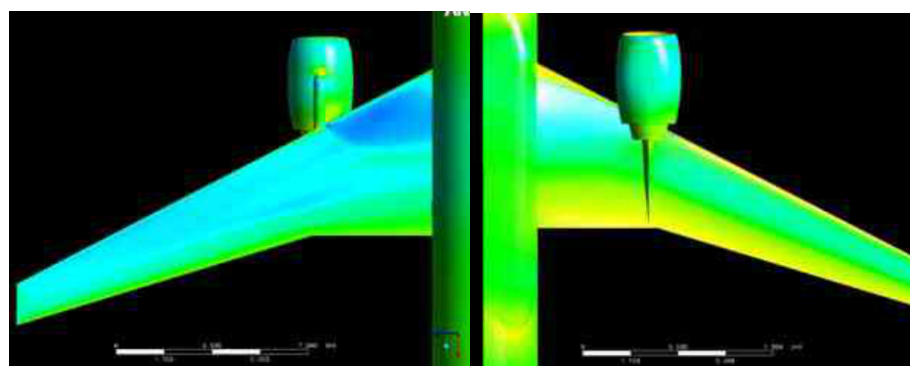

Figure 2.Pressure distribution of the AUV's wings (Boller C 2003) model of aircraft wings has obtained.

As we can see from the graph, the top of the wing and the bottom of the wing experiences high pressure. Therefore, this part of the wing should be monitored carefully.

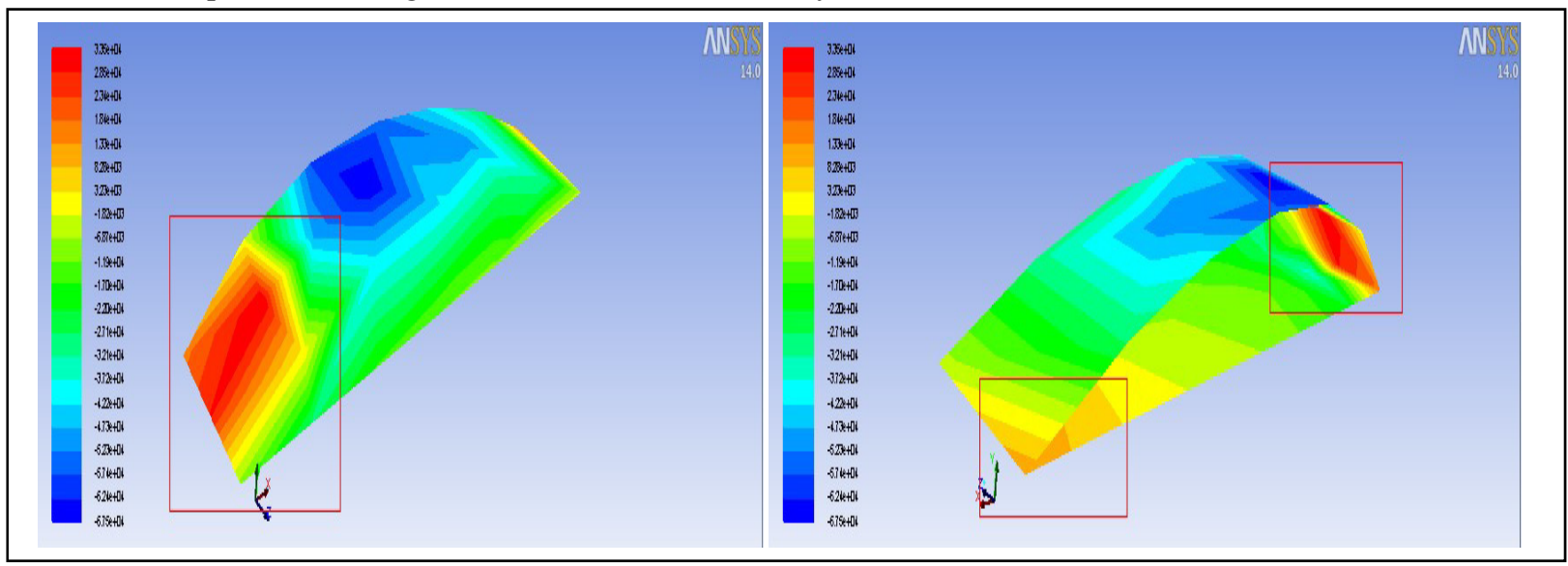

Figure 3.The pressure distribution of modeled wing (Red part experiences high pressure)

Lot of research has been done in relation to battery capacity monitoring. Battery capacity monitoring generally requires the need to measure parameters such as voltage, current, temperature, and most importantly, to calculate remaining capacity. Current method of monitoring battery capacity includes purpose-built chips or the use of a current sensing component and software analysis (such as in the Paparazzi autopilot system). In this way, the ICMS can determine the remaining useful lifetime of the robot, with respect to operating the sensors and propulsion.

In relation to speed detection, the RPM sensors which can detect the speed of vehicle are built into the thrusters. Then the data of the speed can be collected by Arduino. 
X.Xu, A.Anvar, Intelligent Condition Monitoring System (ICMS) for Unmanned Air Vehicle (UAV), Unmanned Surface Vehicle (USV) and Autonomous Underwater Vehicle (AUV), Robots.

\section{FRAME WORK}

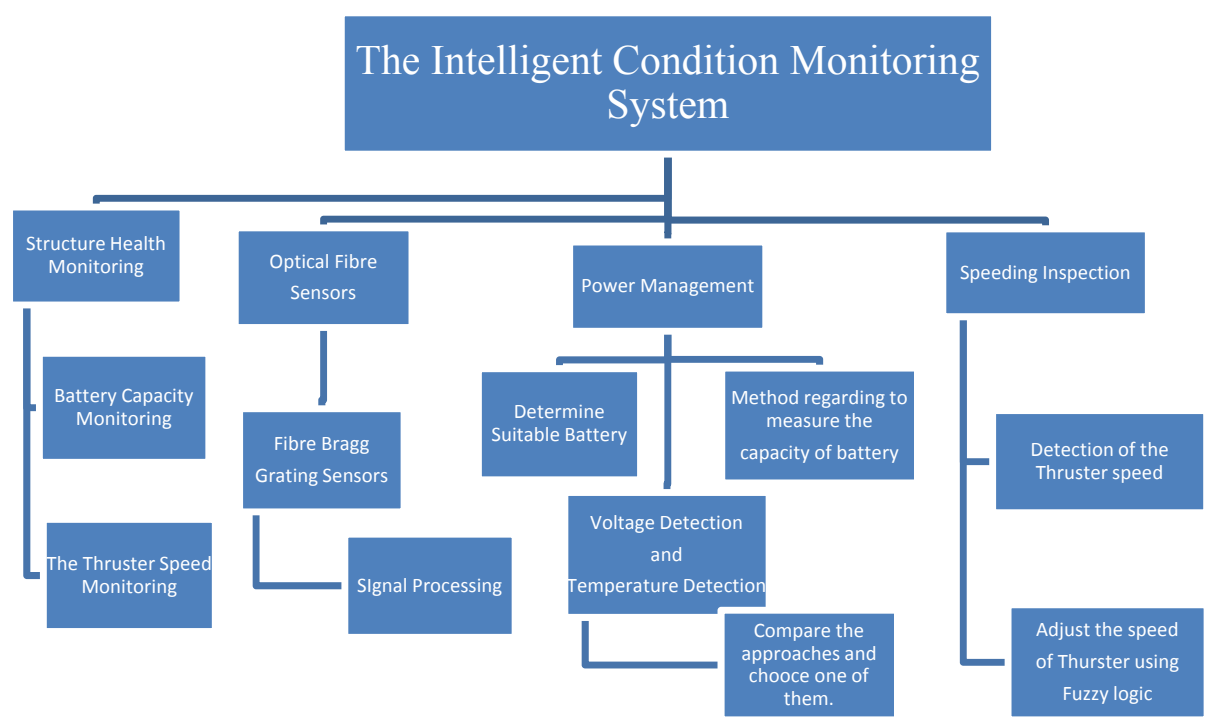

Figure 4. The Frame Work of the ICMS

\section{THE THREE MAIN SECTIONS INCLUDED IN ICMS}

Three main sections are included in ICMS. They are Structure Health Monitoring, Power Management and Speeding Inspection. The three sections can make sure that the vehicles are working in healthy condition.

\section{a) The Structural Health Monitoring System}

Structure health monitoring is important for unmanned vehicles. The vehicles will fail to perform tasks if the structure is damaged. Therefore, the structure needs to be monitored to make sure that the vehicles are working in healthy condition.

We consider use of optical fiber sensors for detecting the status of the structure. The first and most common application of optical fibers is to sense physical parameters such as strain, vibration, temperature and pressure (Foote et al, 2004). Besides, Kosmatka, J. B et al (2007) claimed that the optical fiber sensors are considered for detection of potential damage in UAVs. This includes Operational Load Monitoring (OLM) and impact damage detection system. One of the advantages of using optical fibers is that it can be easily integrated within structures. Moreover, the optical fiber sensors are small in size, which means they can be embedded into a composite structure without significantly degrading their strength (Oliver et al 2007).

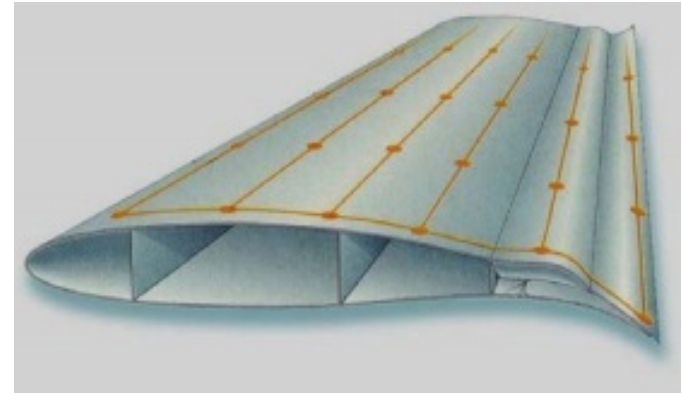

(a) Optical fiber sensors embedded in wing

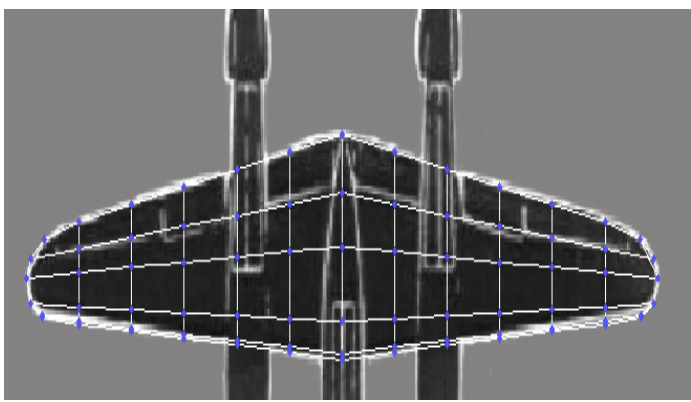

(b) Optical fiber sensors built into UAV

Figure 5.OFS applying in the wing of plane. (Oliver 2007)

When the structure of the wings change, the optical fiber sensor will capture the deformation and send a signal to the intelligent condition system. As we can see from the figure 6 below, normal signal means the 
X.Xu, A.Anvar, Intelligent Condition Monitoring System (ICMS) for Unmanned Air Vehicle (UAV), Unmanned Surface Vehicle (USV) and Autonomous Underwater Vehicle (AUV), Robots.

structure of the wing at this point is healthy (No notch or other damage), if there is a notch on the surface of the wing, the sensors will obtain a signal which is different from the normal signal.

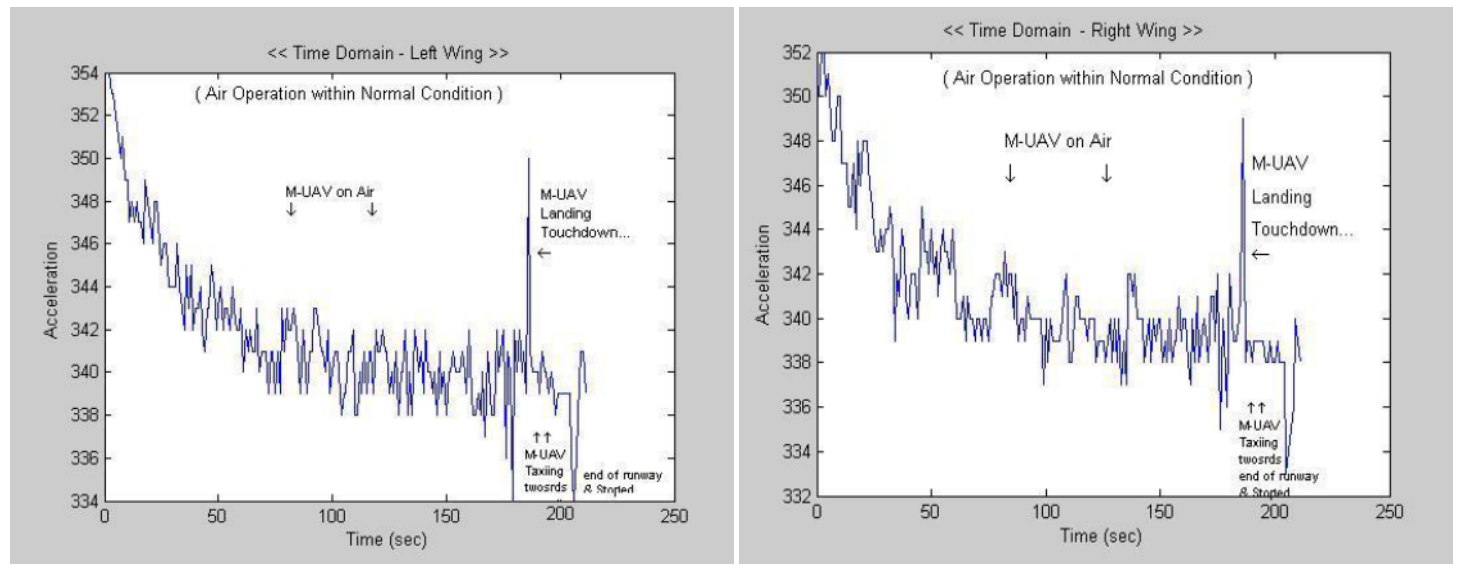

(a) The signal obtained from the left gilder wing

(b) The signal obtained from the right gilder wing
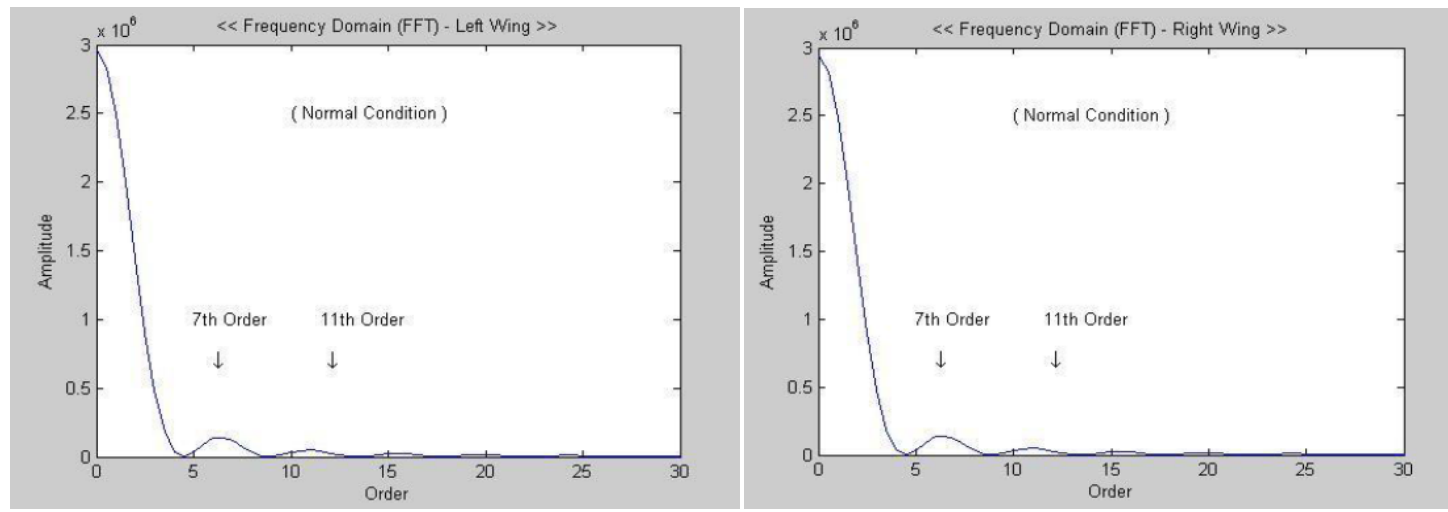

(c) Data processing by using FFT (left wing)

(d) Data processing by applying FFT (right wing)

Figure 6. Signal difference between damaged area and undamaged area.

The curvature damage factor (CDF) can be used in determining the difference among modal curvatures showing damaged wings. In order to calculate the $\mathrm{CDF}$, the equations below are used.

$$
v_{i, j}=\frac{\emptyset_{(i+1), j}+2 \emptyset_{i, j}+\emptyset_{(i-1), j}}{h^{2}}
$$

Where, $v_{i, j}$ : Mode Curvature

i: Node Number

j: Mode Number

h: Distance among Nodes

Then, we need to subtract the undamaged mode shape curvature vector from the damaged component vector:

$$
\Delta v_{i, j}=v_{i, j}^{d}-v_{i, j}^{u}
$$

The change in relation to mode shape curvature is given as:

$$
\Delta v_{i, j}=\left[1+\frac{\Delta v_{i, j}}{\max \left(\Delta v_{i, j}\right)-\min \left(\Delta v_{i, j}\right)}\right]^{2}
$$

Eventually, the ith node for the first $\mathrm{N}$ modes is obtained

$$
C D F_{i}=\frac{1}{N} \sum_{j=1}^{N} \Delta v_{i, j}
$$

Therefore, different combinations of the damage levels at five different locations allow different sets of CDF that are used to create the rules for damage detection. By comparing the values of the training data and 
X.Xu, A.Anvar, Intelligent Condition Monitoring System (ICMS) for Unmanned Air Vehicle (UAV), Unmanned Surface Vehicle (USV) and Autonomous Underwater Vehicle (AUV), Robots.

recorded data, the damage detection can be determined as follows:

\begin{tabular}{|c|c|c|}
\hline Undamaged & No Action & \\
\hline Slight Damage & Advisory Level I & Maintenence Action \\
\hline Moderate Damage & Advisory Level II & \\
\hline Severe Damage & Alert & \\
\hline Damage Level & Warning Level & \\
\hline
\end{tabular}

Figure 7. Schematic Representation of Damage Detection System, Chandrashekhar and Ganguli (2009)

By comparing the severity of fault we can act accordingly to ensure that the structural integrity of the system can be determined.

\section{b) Power Management System}

There are a number of definitions to describe the battery management system depending on the application. A typical definition of battery management system is keeping detection on important parameters such as load voltage, current and battery temperature while operating tasks. Normally, this system would equip the function of protection that can disconnect the battery if there are potential risks.
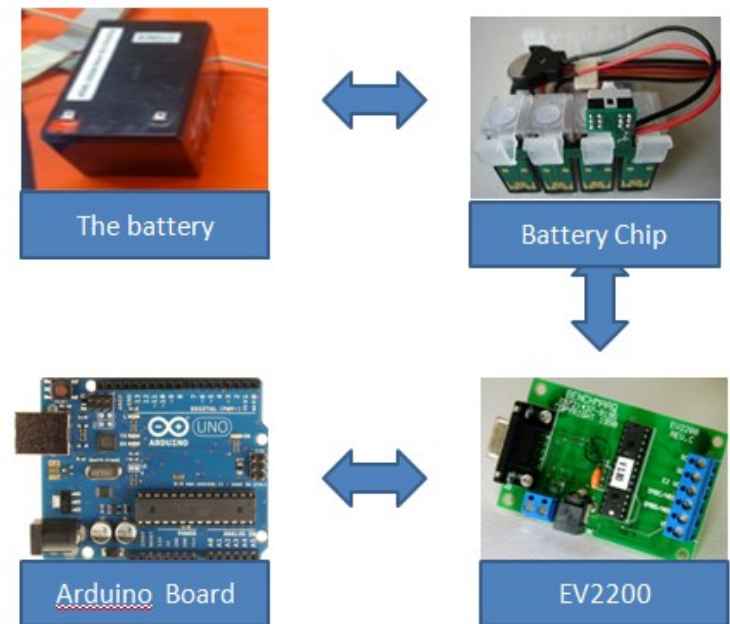

Figure 8. The process of communicating with the Evaluation Module and vice-versa

In order to detect the capacity of batteries, the battery should be connected to a battery chip. The function of the battery chip is to monitor and evaluate the different properties of batteries used. The BQ2013 battery chip is connected to EV2200. The EV2200 acts as A/D converter. The digital signal is then sent to PC for reading.

The measurement and calculation within the BQ2013 is performed by the circuit module which is the DM2013H. The DM2013H evaluates the batteries properties. All this information is needed for fault detection of batteries. The properties of batteries include the battery capacity, the output of current and voltage and the rate of discharge.

The Fuzzy Logic Rules, involved in the Power Management System are as follows:

\begin{tabular}{|c|c|c|}
\hline \multirow{3}{*}{ Fuzzy Logic Rules } & IF: $\mathrm{P}_{\text {Battery }}>\mathrm{P}_{\text {required }}$ & $\begin{array}{c}\text { THEN: Battery status = Excellent } \\
\text { LED GREEN }\end{array}$ \\
\hline & IF: $P_{\text {Battery }}=P_{\text {required }}$ & THEN: Battery status = Average \\
\hline & IF: $\mathrm{P}_{\text {Battery }}<\mathrm{P}_{\text {required }}$ & $\begin{array}{c}\text { THEN: Battery status = insufficient } \\
\text { LED RED }\end{array}$ \\
\hline
\end{tabular}


X.Xu, A.Anvar, Intelligent Condition Monitoring System (ICMS) for Unmanned Air Vehicle (UAV), Unmanned Surface Vehicle (USV) and Autonomous Underwater Vehicle (AUV), Robots.

\section{c) Thrusters Speed Detection Monitoring}

Thruster is one of the most important components for AUVs and UAVs as it supplies the driving power for robot them. The SeaBotix BTD150 is a typical thruster used in UAVs. Two or more thrusters could be used in UAVs to provide propulsion. The most common and important types of faults occurring in UAVs are the asynchronous thrusters, which may lead to termination of the mission. Therefore, building sensors into the thrusters is used to detect and diagnose faults.

One approach to achieve synchronization for different thrusters is to use a thruster motor controller. This unit receives and sends all information via Command \& Control $(\mathrm{C} \& \mathrm{C})$. This unit can detect faults that happen in the thrusters as they provide an accurate measure of actual speed. Besides, the unit can diagnose various types of faults such as overheating and stalled motor, etc.

Thruster Neural Network can contribute to the control of different thrusters as well. As we know, neural network is a structure that tries to find out the relationship between data. Also, it can be trained by using the existing data to improve the accuracy. Once trained, a neural network can then produce estimations and simulations of relevant data.

Due to the neural network relying on input values, a program must be developed to obtain the values from the thrusters, and convert into a form which can be accessed by the neural network (Williams et al 2009). Theoretically speaking, the neural network should have as many inputs as possible. The inputs could come from thruster data and the thruster motor controller. Based on the stated reason, the following inputs are chosen: motor speed, current and temperature.

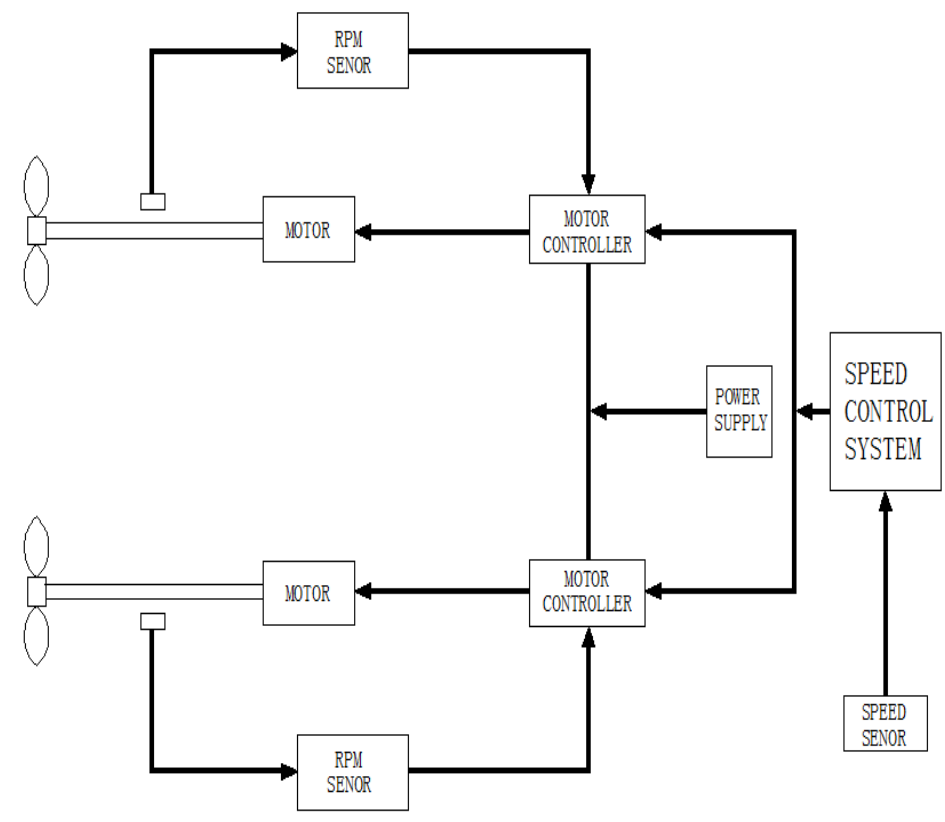

Figure 9. The thrusters monitoring

The speed of the thrusters can be measured by the RPM sensor first. Then the speed control system collects the data of speed, and make sure that the two thrusters are working at the same speed.

\section{CONCLUSION}

An Intelligent Condition Monitoring System is needed in vehicles performing unmanned missions. It detects the status of the structure, inspects the battery power and controlling the speed of the thrusters. The main purpose of the ICMS is to make sure that the vehicles are working in a safe condition. In other words, the ICMS enhances the success rate of performing tasks.

To use optical fiber sensors is a good pathway to detect any damage in the structure of vehicles. However, the limitation is that we have to use much more power to support sensors. In the future research, the critical part of different types of vehicles will be analyzed for reducing the amount of sensors. 
X.Xu, A.Anvar, Intelligent Condition Monitoring System (ICMS) for Unmanned Air Vehicle (UAV), Unmanned Surface Vehicle (USV) and Autonomous Underwater Vehicle (AUV), Robots.

The Fuzzy Logic, as a problem-solving control system methodology, is applied in the system for deciding whether the battery power is sufficient to complete the mission. The disadvantage of using Fuzzy Logic is that if the system is complex, it is hard to use. So, in the future research, the ICMS will contain a simple module rather than a complex one.

For detecting the speed of thrusters, the RPM sensors can be used in ICMS. The speed of thrusters can be monitored by using RPM sensors. Besides, the speed of different thrusters can be controlled by speed control system.

\section{ACKNOWLEDGEMENTS}

The authors would like to acknowledge Defence Science and Technology (DSTO) for the sponsoring the Project. Thanks to several individuals including Kuan Tan for his assistance and support. We appreciate the staff from the School of Mechanical Engineering, Electronics Workshop for their technical assistance and advice. Thanks to the Engineering groups associated with the AUVs and UAVs Projects for their continuous input and assistance in the Project; this was an inspiration to us.

\section{REFERENCES}

Allen B., Stokey R., Austin T., Forrester N., Goldsborough R., Purcell M., von Alt C. (1997). REMUS: A Small Low Cost AUV; System Description, Field Trials, Performance Results. Proceedings of IEEE Ocean 97, 994-1000.

Discalea L.F, et al (2007). Health monitoring of UAV wing skin-to-spar joints using guided waves and macro fiber composite transducers. Journal of intelligent material systems and structures, Vol. 18, no. 4, p.373.

Dowling, T.Putland, T. (2010).Honours Design Project \# 1074 Design , Build and Implementation of Intelligent Troubleshooting Systems for Maritime Air Robots Operations Final Report, 2010 Final Year Report, University of Adelaide.

Kim, Y. (2011). Challenges for rechargeable batteries. Journal of Power Sources, 196(16), pp.6688-6694.

Oliver, J. A., Kosmatka, J. B. (2007). Development of a composite UAV wing test-bed for structural health monitoring research. SPIE Smart Structures and Non-destructive Evaluation Conference, San Diego, CA.

Pusatcioglu Y, Edward G, and Joseph C, 1991, Moisture Sensor', Gas Research Institute, Chicago, US Patent $5,036,704$.

Staszewski W, Boller C \& Tomlinson G.R. (2003) Health Monitoring of Aerospace Structures. Department of Mechanical Engineering, Sheffield University.

Sten G.,P.E. Hagen.,N. Størkersen,K. Vestgård, and K. Per (2002). AUV Based Mine Hunting Demonstrated from MCMV.UDT Europe 2002, La Spezia, Italy.

Tan K.M., T. Liddy, A. Anvar, and L. Tien-Fu (2008). The advancement of an autonomous underwater vehicle (AUV) Technology. Proceedings of Industrial Electronics and Applications, Singapore, 336-341.

Xuri Y., Tommy D., James B. (2000). The application of autonomous underwater vehicles for interdisciplinary measurements in Massachusetts and Cape Cod Bays. Continental Shelf Research, p.p. $2225-2245$. 